\title{
IV. ACYL-COA: 6-APA ACYLTRANSFERASE OF PENICILLIUM CHRYSOGENUM: STUDIES ON SUBSTRATE SPECIFICITY USING PHENYLACETYL-COA VARIANTS
}

\author{
Javier Martin-Villacorta, Angel Reglero \\ and José M. LUENGO* \\ Departamento de Bioquímica y Biología Molecular, Universidad de León, \\ León, España
}

(Received for publication March 30, 1989)

\begin{abstract}
Two different penicillins ( $p$ - and $m$-methylbenzylpenicillin) were obtained "in vitro" by direct enzymatic synthesis, using homogeneously pure acyl-CoA: 6-aminopenicillanic acid (6-APA) acyltransferase from Penicillium chrysogenum, 6-APA and $p$ - or $m$-tolylacetyl-CoA. The $K m$ for these substrates were 6 and $15 \mathrm{~mm}$, respectively, indicating that the affinity of the enzyme for these two molecules is much lower that shown by phenylacetyl-CoA $(0.55 \mathrm{mM})$. Furthermore, acyltransferase does not recognize $o$-tolylacetyl-CoA as a substrate suggesting that the position of the methyl group on the aromatic moiety may have a very important role in the formation of the enzyme-substrate complex.
\end{abstract}

The acyltransferase (AT) of Penicillium chrysogenum catalyses the synthesis of different penicillins on incubating 6-aminopenicillanic acid (6-APA) and different acyl-CoA derivatives ${ }^{1,2)}$. The enzyme recognizes as substrates aliphatic side-chain precursors whose carbon length ranges between 6 and 8 atoms (hexanoic, hexenoic and octanoic acids) suggesting that "in vivo" a single enzyme is able to synthesize benzylpenicillin' and other natural penicillins (DF, F and $\mathrm{K})^{2}$. Further knowledge concerning the specificity of AT has been achieved by testing several variants of the natural substrate (phenylacetyl-CoA) ${ }^{3}$. We have previously reported that this enzyme recognizes as substrates different acyl-CoA derivatives (R-CO-CoA) as long as they maintain an acetyl-CoA moiety in their structure ${ }^{3)}$. Moreover, although the phenyl ring of phenylacetyl-CoA can be replaced by thiophene (2-thiopheneacetyl-CoA), further modifications of the ring (indole acetyl-CoA) or in the acyl-moiety (phenylglycyl-CoA) are not tolerated by $\mathrm{AT}^{3,4}$ ). These findings suggest that the structural requirements for the different acyl-CoA derivatives to be used as substrates by AT are directly related to the acyl chain linked to the acetyl-CoA moiety present in the substrate. In order to shed further light on this point we have studied, in a first approach, the effect that single substitutions in the phenylacetyl moiety of phenylacetyl-CoA cause on the rate and efficiency of 6-APA $N$-acylation. In this report we tested the availability of three different tolylacetyl-CoA derivatives as substrates for $P$. chrysogenum AT.

\section{Materials and Methods}

Materials

$\overline{p-, m-\text { and }} o$-Tolylacetic acids were supplied by Lancaster Synthesis Ltd. (UK). Phenylacetyl-CoA and CoA were obtained from Sigma Chemical (U.S.A.). Benzylpenicillin potassium salts $(1,590 \mathrm{U} / \mathrm{mg})$ were from Antibioticos S.A. León (Spain). $\beta$-Lactamase of Bacillus cereus was from Difco (U.S.A.).

Microorganisms

The strain of $P$. chrysogenum Wis $54-1255$ used in the experimental work was from the American 
Type Culture Collection (ATCC No. 28089). The fungus was grown and cultured as previously described ${ }^{5}$.

Micrococcus luteus ATCC 9341 was used for the determination of the different penicillins by bioassay ${ }^{6}$. The sensitivity of $\beta$-lactam antibiotics to $\beta$-lactamase was established by the lost of their antibacterial activity against $M$. luteus.

\section{Purification of Acyl-CoA: 6-APA AT}

The enzyme was assayed and purified as previously reported ${ }^{1,3)}$.

\section{Synthesis of Acyl-CoA Derivatives}

$p$-, $m$ - and $o$-Tolylacetyl-CoA were synthesized by standard procedures using the acylchloride derivative and coenzyme $\mathrm{A}^{3,7 \sim 9)}$. The transformation yields were higher than $89 \%$ in all three cases.

\section{Results and Discussion}

In order to establish how critical the carbon length of the chain linked to acetyl-CoA is, and the role of its molecular size in the formation of the enzyme-substrate complex, we assayed three different phenylacetyl-CoA variants $(p-, m-$ and $o$ tolylacetyl-CoA) as hypothetical substrates for AT. These molecules (Fig. 1) could mimic rigid structural variants of the aliphatic side-chain present in natural substrates (hexanoyl and octanoyl-CoA) and they might therefore be used as substrates. A similar hypothesis has been proposed by BALDwIN et $a l .{ }^{10)}$ to explain the substrate specificity of isopenicillin N synthase (IPNS) from Acremonium
Table 1. Formation of different penicillins by incubating of AT with 6-APA and different phenylacetyl-CoA derivatives ${ }^{3}$.

\begin{tabular}{lcc}
\hline \multicolumn{1}{c}{ Substrate } & $\begin{array}{c}\beta \text {-Lactam } \\
\text { antibiotics } \\
\text { evaluated as } \\
\text { benzylpenicillin } \\
(\mu \mathrm{g} / \mathrm{ml})\end{array}$ & $\begin{array}{c}\text { Sensitivity } \\
\text { to }\end{array}$ \\
& 0.6 & \\
\hline$p$-TActamase & \\
$m$-TA-CoA $(6 \mathrm{mM})$ & 0.3 & + \\
$o$-TA-CoA $(50 \mathrm{mM})$ & 0 & - \\
Phenylacetyl-CoA $(1 \mathrm{mM})$ & 1.7 & + \\
Idem $+p$-TA-CoA $(1 \mathrm{mM})$ & 1.7 & + \\
Idem $+m$-TA-CoA $(1 \mathrm{~mm})$ & 1.7 & + \\
Idem $+o$-TA-CoA $(50 \mathrm{mM})$ & 1.7 & + \\
\hline
\end{tabular}

$K m$ s calculated for phenylacetyl-CoA, $p$-TA-CoA and $m$-TA-CoA were $0.55,6$ and $15 \mathrm{~mm}$ respectively.

TA: Tolylacetyl.

Fig. 1. Structure of different acyl-CoA derivatives.

I: $o$-Tolylacetyl-CoA, II: $m$-tolylacetyl-CoA, III: $p$-tolylacetyl-CoA; A and B are hypothetical rigid chains of these molecules.

(A)

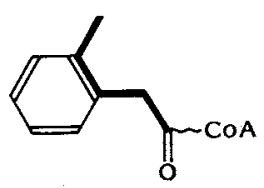

II

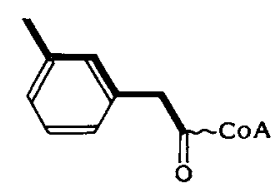

II

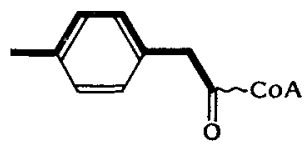

(B)
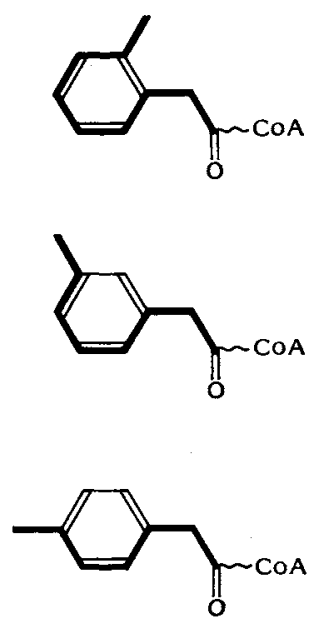
chrysogenum when the $\alpha$-aminoadipolyl moiety of $\delta$-L- $\alpha$-aminoadipyl-L-cysteinyl-D-valine (ACV) is replaced by phenylacetic acid ${ }^{6)}$ and $m$-carboxyphenylacetic acid $^{10}$.

If the 6 to 8 carbon atom length requirements hypothesis is correct, one would expect that the molecules obtained by replacing one of the hydrogen atoms of the phenyl moiety of phenylacetyl-CoA by methyl radicals, could be used as substrates as long as the length of their rigid chains range between 6 and 8 carbon atoms.

The incubation of pure acyl-CoA: 6-APA AT with 6-APA and $p$ - and $m$-tolylacetyl-CoA generated molecules that were active against $M$. luteus ATCC 9341 and sensitive to $\beta$-lactamase but did not with $o$-tolylacetyl-CoA (Table 1). Kinetic studies have shown that the $K m$ values calculated for both substrates ( $p$ - and $m$-methyl derivatives) were 6 and $15 \mathrm{mM}$, respectively. These values, which are much higher than the $K m$ value reported for phenylacetyl-CoA $(0.55 \mathrm{~mm})^{1,3)}$ (Table 1$)$, indicate that the affinity of the enzyme for these compounds is lower than for the natural substrate. Furthermore, when phenylacetyl-CoA and $p$ - or $m$-tolylacetyl-CoA were added together in the reaction mixture, at the same final concentration ( $1 \mathrm{mM}$ ), the efficiency of benzylpenicillin biosynthesis did not decrease (Table 1), suggesting that they do not act as inhibitors of the natural reaction. Similar results were obtained when AT, 6-APA and $o$-tolylacetyl-CoA were incubated with phenylacetyl-CoA. In this case, the rate of 6-APA $\mathrm{N}$-acylation with phenylacetic acid was not affected even when a 50-fold increased concentration of $o$-tolylacetyl-CoA was present (see Table 1). These results suggest that $o$-tolylacetyl-CoA does not bind to the enzyme, reinforcing the importance of the position of the methyl group on the molecule of substrate.

The results shown in this report seem to agree with the AT minimal and maximal side-chain length requirement hypothesis since $p$ - and $m$-tolylacetyl-CoA could represent rigid structures ranging between 6 to 8 carbon atoms in length (see Fig. 1). However, in 0 -tolylacetyl-CoA the length of the rigid chains are of 5 or 9 carbon atoms (Fig. 1). In either orientation (A or B), the size of the acyl-moiety is outside the critical limits required by this enzyme (C-6 to C-8).

On the other hand, the lower affinity of AT for $p$ - and $m$-tolylacetyl-CoA when compared with phenylacetyl-CoA (see above) suggests that although the carbon chain length is very important ${ }^{2)}$, the presence of a methyl group on the molecule of substrate causes steric hindrance.

\section{References}

1) Luengo, J. M.; J. L. Iriso \& M. J. LóPEZ-Nieto: Direct evaluation of phenylacetyl-CoA: 6-Aminopenicillanic acid acyltransferase of Penicillium chrysogenum by bioassay. J. Antibiotics 39: 1565 1573, 1986

2) Luengo, J. M.; J. L. IRISo \& M. J. LóPEZ-NIETo: Direct enzymatic synthesis of natural penicillins using phenylacetyl-CoA: 6-APA phenylacetyl transferase of Penicillium chrysogenum: Minimal and maximal side chain length requirements. J. Antibiotics 39: 1754 1759, 1986

3) Alonso, M. J.; F. Bermejo, A. Reglero, J. M. Fernández-Cañón, G. González de Buitrago \& J. M. Luengo: Enzymatic synthesis of penicillins. J. Antibiotics 41: 1074 1084, 1988

4) Luengo, J. M.: Recent advances in the enzymatic synthesis of penicillins. In Progress in Industrial Microbiology. Vol. 27. Eds., M. E. Bushell \& K. Grafe, pp. 315 332, Elsevier, 1989

5) Luengo, J. M.; G. Revilla, J. R. Villanueva \& J. F. Martin: Lysine regulation of penicillin biosynthesis in low-producing and industrial strains of Penicillium chrysogenum. J. Gen. Microbiol. 115: 207 211, 1979

6) Luengo, J. M.; M. T. Alemany, F. Salto, F. R. Ramos, M. J. López-Nieto \& J. F. Martín: Direct enzymatic synthesis of penicillin $\mathrm{G}$ using cyclases of Penicillium chrysogenum and Acremonium chrysogenum. Bio/Technology 4: $44 \sim 47,1986$

7) BeEBy, P. J.: Angelolyl chloride: Synthesis and utilisation in the partial synthesis of lantadene A (rehmannic acid). Tetrahedron Lett. 1977: 3379 3382, 1977

8) Wolfe, S.; J. C. Godfrey, C. T. Holdrege \& Y. G. Perron: Rearrangement of penicillins to anhydropenicillins. 
Can. J. Chem. 46: 2549 2559, 1968

9) Fleet, G. W. J. \& P. J. C. HARding: Convenient synthesis of bis(triphenylphosphine) copper(I) tetrahydroborate and reduction of acid chlorides to aldehydes. Tetrahedron Lett. 1979: 975 978, 1979

10) Baldwin, J. E; R. M. Adlington, M. James, C. Crabbe, C. G. Knight, T. Nomoto \& C. J. Schofield: An effective substitute for $\alpha$-aminoadipic acid in the enzymatic synthesis of penicillins. J. Chem. Soc. Chem. Commun. 1987: $806 \sim 807,1987$ 